\title{
A REMARK ON $A_{1}$-WEIGHTS FOR THE STRONG MAXIMAL FUNCTION
}

\author{
FERNANDO SORIA
}

\begin{abstract}
If $g$ is a locally integrable function and $M$ is the Hardy-Littlewood maximal function then $(M g)^{\delta}$ represents an $A_{1}$-weight of $M$ for every $0<\delta<$ 1; that is, $M(M g)^{\delta}(x) \leq C_{\delta}(M g(x))^{\delta}$ a.e. In this paper we show that this result does not hold in general if we replace $M$ by the strong maximal operator.
\end{abstract}

Given an operator $T$ defined on some subclass $X$ of the set of measurable functions in $\mathbf{R}^{n}$ we say that $w \in X$ is an $A_{1}$-weight for $T$ if

$$
|T w(x)| \leq C w(x) \quad \text { a.e. }
$$

where $C$ is a constant which depends on $w$. We denote the class of all $A_{1}$-weights of $T$ by $A_{1}(T)$. (See Muckenhoupt [3].)

The theory of weights has been very valuable in the study of many operators in classical harmonic analysis. Among others, the following result has been particularly important since it gives a description of the class $A_{1}(T)$, when $T=M$ is the Hardy-Littlewood maximal operator (i.e., the operator given by the supremum on the averages on cubes which contain a fixed point).

Let $g$ be locally integrable and so that $M g(x)<\infty$, a.e. Then $w=(M g)^{\delta} \in$ $A_{1}(M)$ for every $0 \leq \delta<1$. (See Coifman and Rochberg [1].) Moreover, as an easy consequence of the reverse Hölder inequality one also has a converse statement. Namely, if $w \in A_{1}(M)$, there exist $h(x)$ in $L_{\text {loc }}^{1}, \delta>0$, and $k(x)$ with $k$ and $1 / k \in L^{\infty}$, such that $w(x)=k(x)(M h(x))^{\delta}$. So that, indeed, all $A_{1}$-weights of $M$ are essentially of the form $(M g)^{\delta}$.

In this paper we show that the first part of the above result does not hold in general if we replace $M$ by the strong maximal operator $M_{S}$ (averages on "intervals"). The study of whether or not this could be the case was proposed to the author by J. L. Rubio de Francia, to whom we are indebted. The question appears in [2] as an open problem.

We state now the main result. Further generalizations to the setting of product domains are given below.

PROPOSITION. There exists $g \in \bigcap_{0<p \leq \infty} L^{p}\left(\mathbf{R}^{n}\right)$ with the following property. For every $0<\delta$ and every constant $C>0$, there is a set $S$ of positive measure such that

$$
M_{S}\left(M_{S} g\right)^{\delta}(x) \geq C\left(M_{S} g\right)^{\delta}(x), \quad \forall x \in S .
$$

Indeed, inequality (1) holds on a set of infinite measure.

Received by the editors February 28, 1986.

1980 Mathematics Subject Classification (1985 Revision). Primary 42B25.

Key words and phrases. Maximal operators, weights, product domains.

Work supported in part by a grant of the NSF.

(C) 1987 American Mathematical Society $0002-9939 / 87 \$ 1.00+\$ .25$ per page 
For simplicity we will only consider here the case $n=2$, the other cases being similar. This proposition is an easy consequence of the following.

LEMMA. $\forall N \in \mathbf{N}$, there exists $v=v_{N}$ with compact support so that

$$
\left\{M_{S}\left(M_{S} v\right)^{\delta}(x)\right\}^{1 / \delta} \geq c_{\delta} N M_{S} v(x), \quad \forall x \in[0,1]^{2} .
$$

Moreover,

$$
\left\|v_{N}\right\|_{L^{p}} \leq c_{p} 2^{N}, \quad \text { for } 0<p \leq \infty .
$$

In order to see how this lemma implies the above result, one can just consider the sequence of functions $\left\{v_{N}\right\}$ whose existence is ensured by the lemma and form the function

$$
g(x)=\sum 2^{-2 N} v_{N}\left(x-x_{N}\right)
$$

where the $x_{N}$ 's are chosen very distant one from another in such a way that the values of $M g$ on the square $\left[x_{N}, x_{N}+1\right]^{2}$ are the same as the values of $M\left(2^{-2 N} v_{N}\right)$ on $[0,1]^{2}$. This can be easily done due to the compactness of the support of the $v_{N}$ 's. In fact, a simple analysis of the constants appearing in the proof of the lemma shows that $X_{N}=\left(2^{N}, 2^{N}\right)$ would do it.

ProOF of THE LEMMA. Given $k, j \in \mathbf{Z}$, let $Q_{k, j}$ denote the unit square with lower left corner at the point $(k, j)$. For $k=1,2, \ldots, N$ let $d_{k}$ be the integral part of $2^{k} / k$ and set

$$
v(x)=v_{N}(x)=\sum_{1 \leq k \leq N} 2^{k} \chi_{Q_{k, d_{k}}}(x) .
$$

Then clearly $\|v\|_{\infty}=2^{N}$ and $\|v\|_{L^{p}}=\sum_{1}^{N} 2^{k p}$ for $0<p<\infty$. Therefore, (2) holds with $c_{p}=2\left(2^{p}-1\right)^{-1 / p}$.

Now, if $R_{k}$ is the rectangle $[1, k+1] \times\left[1, d_{k}+1\right]$, a simple computation shows that $\forall x \in[0,1]^{2}$ we have

$$
M_{S} v(x) \leq M_{S} v((1,1))=\sup _{1 \leq k \leq N}\left|R_{k}\right|^{-1} \int_{R_{k}} v(y) d y \leq 2 .
$$

On the other hand, if $\tilde{R}_{k}=[k, k+1] \times\left[1, d_{k}+1\right]$ then for every $x \in \tilde{R}_{k}$ we have

$$
M_{S} v(x) \geq\left|\tilde{R}_{k}\right|^{-1} \int_{\tilde{R}_{k}} v(y) d y=2^{k} / d_{k} \geq k / 2 .
$$

Hence, $f=M_{S} v(x) \geq k / 2$ on $Q_{k, j}$ for $j=1,2, \ldots, N$ provided that $d_{k} \geq N$ (e.g., if $k \geq 2 \log _{2} N$ ).

Finally, if we take the square $R=[0, N+1] \times[0, N+1]$ we obtain for every $x \in[0,1]^{2}$

$$
\begin{aligned}
{\left[M_{S}\left(M_{S} v\right)^{\delta}(x)\right]^{1 / \delta} } & \geq\left[M_{S}(f)^{\delta}((0,0))\right]^{1 / \delta} \\
& \geq\left(|R|^{-1} \int_{R}(f)^{\delta} d y\right)^{1 / \delta} \\
& \geq c\left[(N+1)^{-2} \sum_{2 \log _{2} \leq k \leq N} N(k)^{\delta}\right]^{1 / \delta}=O(N)
\end{aligned}
$$


This finishes the proof of the lemma and, therefore, of the above proposition. Q.E.D.

REMARKS. (1) The fact that in the last stage of the previous proof we have considered a square shows that the function $w=\left(M_{S} g\right)^{\delta}$ is not even an $A_{1}$-weight for the Hardy-Littlewood maximal operator. This is stronger than $w \notin A_{1}\left(M_{S}\right)$ since $M f \leq M_{S} f \forall f$ and, therefore, $A_{1}\left(M_{S}\right) \subset A_{1}(M)$.

(2) One could try to produce $A_{1}$-weights for the operators arising in product domains as follows: Let $M_{i}, i=1,2$, be the 1-dimensional Hardy-Littlewood operator in the $x_{i}$-direction. Given $f \in L_{\text {loc }}^{1}$ we consider $\left(M_{2} M_{1} f\right)^{\delta}$ for some $\delta<1$ and we ask whether this is necessarily a weight in $A_{1}\left(M_{2} \circ M_{1}\right)$. The answer is no and the function $g$ is again a counterexample.

In fact, the weaker statement $M_{1}\left(M_{2} f\right)^{\delta} \leq C\left(M_{2} M_{1} f\right)^{\delta}$ a.e. is not true in general. For if we take $N \in \mathbf{N}$ and $v=v_{N}$ as in the lemma then

$$
M_{2} M_{1} v(x) \sim 1 \quad \text { on } Q_{1,1}=[1,2]^{2},
$$

whereas

$$
\left(M_{1}\left(M_{2} v\right)^{\delta}(x)\right)^{1 / \delta} \sim N \quad \text { on } Q_{1,1} .
$$

Perhaps it is interesting to notice here that the inequality

$$
M_{2}\left(M_{1} f\right)^{\delta} \leq\left(M_{2} M_{1} f\right)^{\delta} \quad \forall f
$$

is trivial for $0<\delta \leq 1$, by Jensen's inequality.

(3) Another possible way of constructing $A_{1}$-weights in the setting of product domains could be to look at $\left(M_{2}\left(M_{1} f\right)^{\delta}\right)^{\mu}, 0<\delta, \mu<1$, for some $f \in L_{\text {loc }}^{1}$. Again, this is not the case. To see this, it suffices to consider $f(x)=(g(x))^{1 / \delta}$, for $0<\delta<1$ fixed.

A simple computation shows that $\left(M_{2}\left(M_{1}\left(v_{N}\right)^{1 / \delta}\right)^{\delta}\right)^{\mu} \sim N^{(1-\delta) \mu}$ on $Q_{1,1}$, whereas from remark (2) one has

$$
M_{1}\left(M_{2}\left(M_{1}\left(v_{N}\right)^{1 / \delta}\right)^{\delta}\right)^{\mu} \geq M_{1}\left(M_{2} v_{N}\right)^{\mu} \sim N^{\mu} \quad \text { on } Q_{1,1} .
$$

Therefore, $w=\left(M_{2}\left(M_{1} f\right)^{\delta}\right)^{\mu} \notin A_{1}\left(M_{1}\right)$ (hence $w \notin A_{1}\left(M_{S}\right) \cup A_{1}\left(M_{1} \circ M_{2}\right) \cup$ $\left.A_{1}\left(M_{2} \circ M_{1}\right)=A_{1}\left(M_{S}\right)\right)$. However, $w$ does belong to $A_{1}\left(M_{2}\right)$, from Coifman and Rochberg's result.

\section{REFERENCES}

1. R. Coifman and R. Rochberg, Another characterization of BMO, Proc. Amer. Math. Soc. 79 (1980), 249-254.

2. J. Garcia-Cuerva and J. L. Rubio de Francia, Weighted norm inequalities and related topics, North-Holland Math. Studies, vol. 116, North-Holland, Amsterdam, 1985.

3. B. Muckenhoupt, Weighted norm inequalities for the Hardy maximal function Trans. Amer. Math. Soc. 165 (1972), 207-226.

Department of Mathematics, University of Chicago, Chicago, Illinois 60637

Current address: Universidad Autónoma de Madrid, Matemáticas, 28049 Madrid, Spain 